\title{
Understanding Food Preferences and Their Connection to Health Perception among Lean and Non-Lean Populations in a Rural State
}

Mark Menghini, BS; Reshmi Singh, BPharm, MS, PhD; Baskaran Thyagarajan, MPharm, PhD

School of Pharmacy, University of Wyoming

\begin{abstract}
Lipid metabolism and dietary choices directly affect the outcome of overall weight management in both lean and non-lean individuals. However, the perception of consuming spicy foods has diverse meaning among people. To understand this, it is essential to have thorough knowledge on how food preference is tied to health outcomes. The aim of this study is to enhance the understanding of how food preference affects the health outcome and perception in lean and non-lean populations. A mixed methods study was conducted via analysis of consumers' food choices and compared the data based on age, gender, and body weight. The participants in audio recorded interviews were comprised of residents from a single town in a rural state. The study shows that most participants were aware of the implications that food choices had on their health status and it emphasizes the importance of understanding the differences between consumption of spicy and non-spicy foods. Spicy food consumption was associated with decreased overall portion size as well as increased satisfaction following the meal. Environmental factors, such as the influence of family and friends, impacted spicy food consumption according to most participants. The outcome of the study provides a comprehensive understanding of food preferences from a relatively large exploratory study. The observations made here show rudimentary associations between physical attributes and levels of food consumption. Future studies could further identify how certain attributes relate to food choices and levels of spicy food consumption in greater detail.
\end{abstract}

Keywords: Spicy food, food preference, lean, non-lean, population study, qualitative analysis

\section{INTRODUCTION}

Obesity is a critical cardiac risk factor that predisposes to serious health complications such as diabetes, hypertension, and myocardial infarction (Singh et al., 2017). Although a combination of lifestyle changes, exercise, pharmacotherapy, and surgical interventions help in obesity management, obesity rates continue to increase, and approximately $34 \%$ of adults and $20 \%$ of children in the United States are obese (Mitchell et al., 2011). This translates into high costs for treating illnesses related to obesity and poses tremendous burden on the healthcare system (Singh et al., 2017). The development of a reliable therapeutic strategy to target obesity could begin to help reverse the issue. Published work from our laboratory suggest that pungent capsaicin, an active ingredient in chili peppers, is more effective than non-pungent analogs in preventing diet-induced obesity (Baskaran et al., 2018). Capsaicin, the chemical that gives peppers its spiciness, has been shown to stimulate increased energy expenditure and fat oxidation to reduce body fat (Bloomer et al., 2010; Caterina et al., 1997; Haramizu et al., 2011; Haramizu et al., 2011; Janssens et al., 2013; Ludy \& Mattes, 2011; Ludy, Moore, \& Mattes, 2012; Nirengi, 2016; Ohnuki et al., 2001; Rondanelli et al., 2013). A combination of lipid metabolism, dietary preference, and energy expenditure directly affect the outcome of overall

Corresponding author: Mark Menghini, BS

School of Pharmacy, University of Wyoming

1000 E University Ave, Laramie, WY 82071

Email: $\underline{\text { mmenghi1@uwyo.edu }}$ weight in which individuals may be categorized as lean or nonlean via the Body Mass Index (BMI) scale. It has been found recently that the consumption of spicy food is inversely related to LDL- cholesterol levels which further supports the need for a large-scale, survey-based study to be completed (Xue et al., 2017).

Assuming no presence of extreme social pressures influencing food choice, it has been found that taste is the primary force that drives food preference while the choice for healthy food follows next (Eertmans, Baeyens, \& Van den Bergh, 2001; IFIC, 2014). Of those with restricted budgets due to socioeconomic factors, preference for healthy food may not always be a viable choice. Surveying people on the reasoning behind their food choices may lead to open ended responses that further explain the complexities of decisions regarding food for themselves and their families. The major contributor for the overall satisfaction often seems to revolve around portion size. However, spicy food may also play a significant role in providing enough stimulation to satisfy the consumer during or after a meal.

Considering the fact that the perception of spicy food widely varies among people, reviewing the attributes that people have may explain the differences in liking or disliking spicy foods (Byrnes \& Hayes, 2015). One significant factor is past exposure to spicy food on a regular basis (Ludy \& Mattes, 2012). Cultural characteristics and family habits play a huge role in peoples' food choices, especially in the case of spicy versus non-spicy foods. Repeated exposure to capsaicin containing foods has been shown to induce a developed tolerance to spiciness and people can even learn to enjoy the sensation (Cowart, 1981; 
Green \& Hayes, 2003; Karrer \& Bartoshuk, 1991; Lawless, Rozin, \& Shenker, 1985; Stevenson \& Prescott, 1994; Stevenson \& Yeomans, 1995). Naturally, those who become accustomed to the sensations of spicy food may find non-spicy food to be bland and unsatisfying; therefore, the group can be thought of as sensation seekers. Sensation seekers can find thrill in many things including the use of spicy substances and eating foods that have peppers which contain capsaicin (Logue \& Smith, 1986; Rozin \& Schiller, 1980). The sensation seekers often allow themselves to create an experience in which they feel an increased satisfaction in a relatively mild and controlled manner compared to more extreme and possibly dangerous activities. In general, people who are looking to gain a unique experience are of a certain personality construct, which certainly affects food choice (Byrnes \& Hayes, 2013). In past research, a sensation seeking scale has been created to quantify the optimal stimulation level (Zuckerman et al., 1964). This analysis did not utilize the previously created sensation seeking scale, but instead guided the participants' open-ended responses via particularly phrased questions designed specifically for this study.

Dietary preferences were assessed in this qualitative study to develop a basic understanding on everyday choices surrounding diet and how they relate to certain personal attributes. Specifically, the difference in spicy food consumption among lean and non-lean participants was explored. Literary searches did not identify any studies that examined consumer perception of lean and non-lean populations on intake of spicy food or the impact spicy food has on overall portion size and satisfaction. The driving forces of food choice, portion size, overall satisfaction, and spicy food intake were examined closely to determine if these various preferences associated with consumer attributes.

\section{MATERIAL and METHODS Overview}

Concurrent quantitative and qualitative analysis of consumer's food choices of different age, gender, and body weight were completed. Audio recordings of face to face, individual interviews led to the creation of verbatim transcripts which were then organized using the qualitative analysis software NVIVO $^{\mathrm{TM}}$ (Appendix, Figure A.2). The interviewees were comprised of residents from a single town in a rural state and were of different age, gender, and lean and non-lean status to ensure diverse information that was used to determine trends and perceptions that affected food preferences. The qualitative aspect of the study was performed via analysis of the participants' open-ended responses in the form of quotations whereas the quantitative portion of the study was performed via calculating the percentage of participants that expressed given attributes compared to their placement in categories regarding their various food preferences.

\section{Participants}

The study included lean (BMI $<24 \mathrm{~kg} / \mathrm{m}^{2}$ ) and non-lean (BMI > $25 \mathrm{~kg} / \mathrm{m}^{2}$ ) participants, aged between 21 and 75 years of age who live, work, or study in a small town in Wyoming. There were 29 lean participants and 31 non-lean participants which allowed answers to be covered in a large spread relative to both attributes. Overall, 14 males and 46 females were recruited through flyers and in person recruitment at libraries, community pharmacies, medical and dental offices, grocery stores, places of worship, a community recreation center, and across the University Campus. This study was approved by the University Institutional Review Board.

\section{Analysis}

Data analysis was then quantified to represent groups using spreadsheets in Microsoft Excel software. Quantitative results were represented via percentages of the total number of 60 participants or of subgroups created based on participant attributes. Keywords and categories were created to place the participants into groups for these quantifications. Z-scores and corresponding $p$-values were calculated to determine significance using Microcal Origin 6.0 software. Qualitative analysis used a descriptive approach to allow for content and quotes to emerge that fit the broad themes of the study (Appendix, Figure A.1).

\section{RESULTS}

\section{Difference in terminology is crucial}

The study requires understanding the differences between spicy food consumption versus non-spicy food consumption. Data from the study revealed the lack of understanding from the participants on the difference between "hot" and "spicy" foods. When asked if "spicy" food is the same as "hot" food, $53.33 \%(z=0.516, p=0.3029272)$ responded that it was not meant to have the same meaning. With exactly $50.00 \%(z=0$, $\mathrm{p}=0.5$ ) being both lean and non-lean. Interestingly, 27 participants were quoted saying it was the same and $88.89 \%$ $(z=1.50, p=0.0668072)$ of those responses were recorded from females with only $11.11 \%(z=-1.50, p=0.0668072)$ being male. "I think of spicy when I think of hot." (Subject 12). A total of $51.67 \%$ of participants stated that they consumed some amounts of spicy food, but it certainly did not make up the majority of their diet. Whereas $40.00 \%$ of participants consumed spicy food often enough to considered it a major part of their diet by having it several times a week and even up to everyday, $66.67 \%(z=-1.16, p=0.1230244)$ were female.

\section{Healthy ingredients and cost are the two primary drivers of food choice in the majority of participants}

The study showed that most participants were aware of the implications that their food choices had on their health status. The initial question asked to each participant was to describe the most important factor in their choice when selecting food items and $45.00 \%$ of participants stated that their primary concern was nutritional value and/or how healthy the food was considered. It was found that $59.26 \%(z=0.789, p=0.215056)$ of 
those 27 participants who selected food based on healthfulness were non-lean. Of all 29 lean participants asked this question, 94.00\% ( $z=4.740, p=0.0000011)$ understood the way diet relates to health. Some non-lean participants struggled to understand the correlation between diet and their health standing regarding BMI. Contrarily, of the $26.67 \%$ of participants who stated that taste was the primary factor concerning food choice, $50.00 \%(z=0.134, p=0.4467013)$ were lean and $50.00 \%(z=-0.134, p=0.4467013)$ were non-lean.

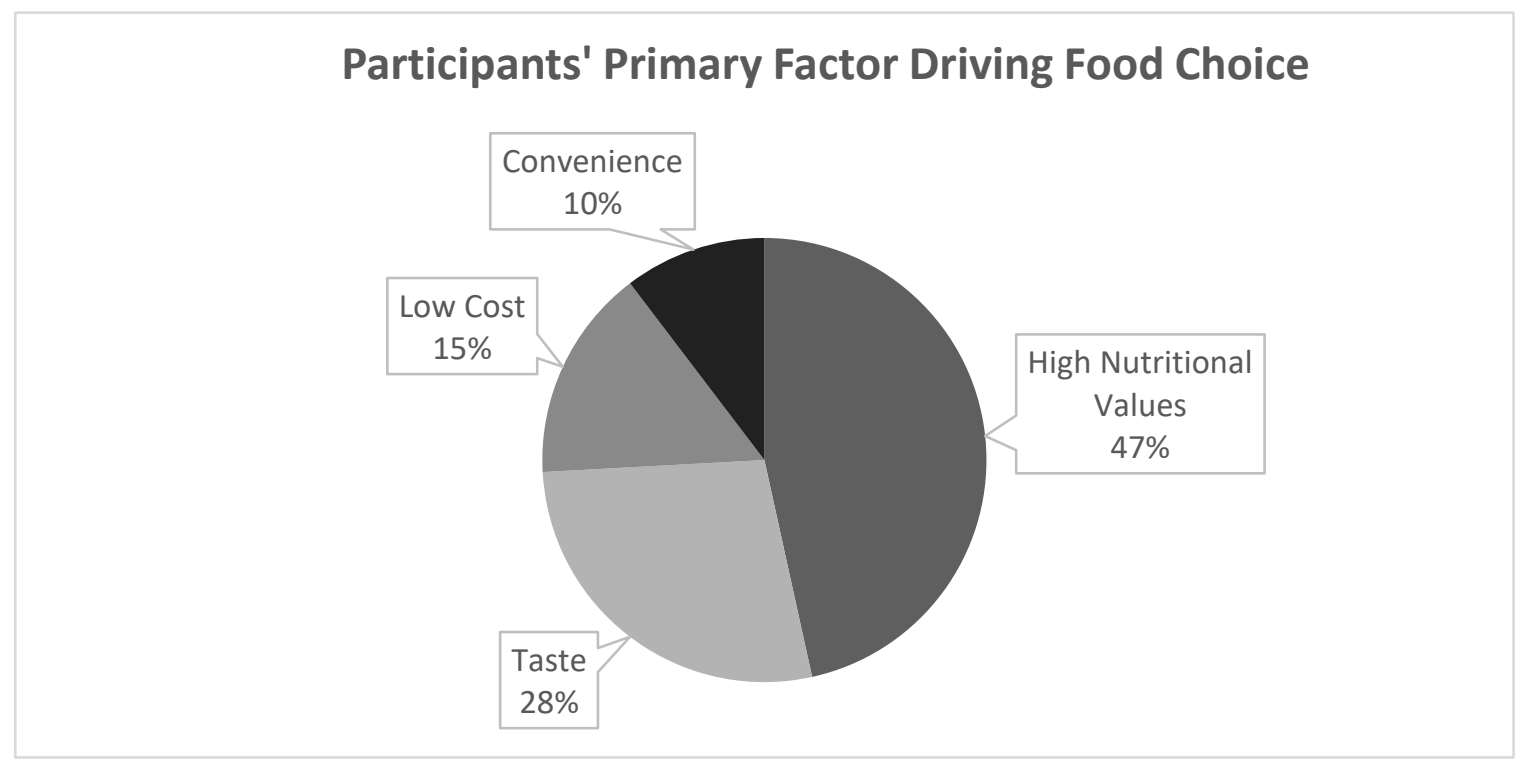

Figure 1. Pie chart represents the percentage of total survey population who stated the primary driver in food choice was either high nutritional values, preferential taste, low cost, or convenience.

When eating spicy food, the majority of participants expressed greater satisfaction and decreased overall consumption compared to non-spicy foods

Table 1. Quotations directly from interviewed participants with

regards to greater overall satisfaction and decreased overall consumption.

\begin{tabular}{|c|l|}
\hline Subject Number & \multicolumn{1}{c|}{ Quotation from Transcript } \\
\hline 47 & $\begin{array}{l}\text { "I think I can feel fuller faster when I'm eating spicy hot foods then when I'm just eating } \\
\text { sort of, I'm going to say a bland meal." }\end{array}$ \\
\hline 42 & "I like spicy food, so I tend to feel more satisfied after spicy." \\
\hline 24 & "I think that I might eat a little less of the spicy food." \\
\hline
\end{tabular}

Of all 60 participants, $71.67 \%$ defined "hot" in the context of food to mean spicy with $81.40 \%(z=0.733, p=0.2317792)$ of those responses from female participants. Some participants (15.00\%) stated temperature was intended when speaking of "hot" in the context of food while another $11.67 \%$ concluded that either spiciness or temperature could be meant by "hot".

When the participants were asked about the effects spicy food had on them, the most common response was because it burned and was irritating which consisted of $33.33 \%$ of all participants who preferred to not consume spicy food. Another $20.00 \%$ stated that it was warm and not preferred. A smaller group of $15.00 \%$ of the participants stated it was the taste of spicy food they did not find enjoyable as well as $15.00 \%$ who find spicy food simply unpleasant. Leaving only $8.33 \%$ of participants to find the stimulus pleasant. Of those who found the stimulus pleasant, $80.00 \% \mathrm{z}=0.176, p=0.430147)$ were female and $60.00 \%(z=0.373, p=0.3545742)$ were non-lean. $A$ large group consisting of $63.33 \%$ of participants stated that spicy food made a difference in their sense of satisfaction with $76.32 \%(z=-0.051, p=0.4796628)$ being female and $57.89 \%$ $(z=0.767, p=0.2215408)$ were categorized as non-lean.

When spicy food was served, $51.67 \%$ stated that it negatively affected the amount of total food consumed due to being spicy compared to $11.67 \%$ of participants who stated that they would eat more spicy food if it were served. While $31.67 \%$ said it had no effect on the amount of either spicy or non-spicy food consumed. Interestingly, $58.06 \%(z=0.712, p=0.2382324)$ of the people who eat less spicy food when served were non-lean and $41.94 \%(z=-0.712, p=0.2382324)$ were lean. 


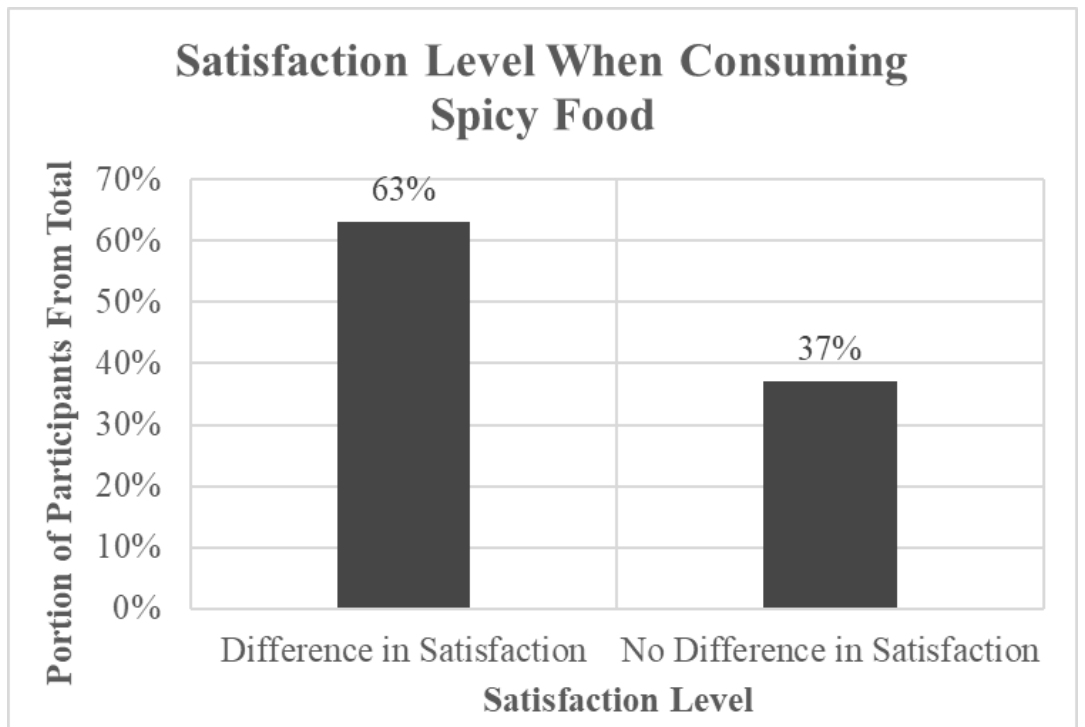

Figure 2. Bar graphs represent the percentage of total survey population who either found a difference or no difference in satisfaction after consuming spicy foods.

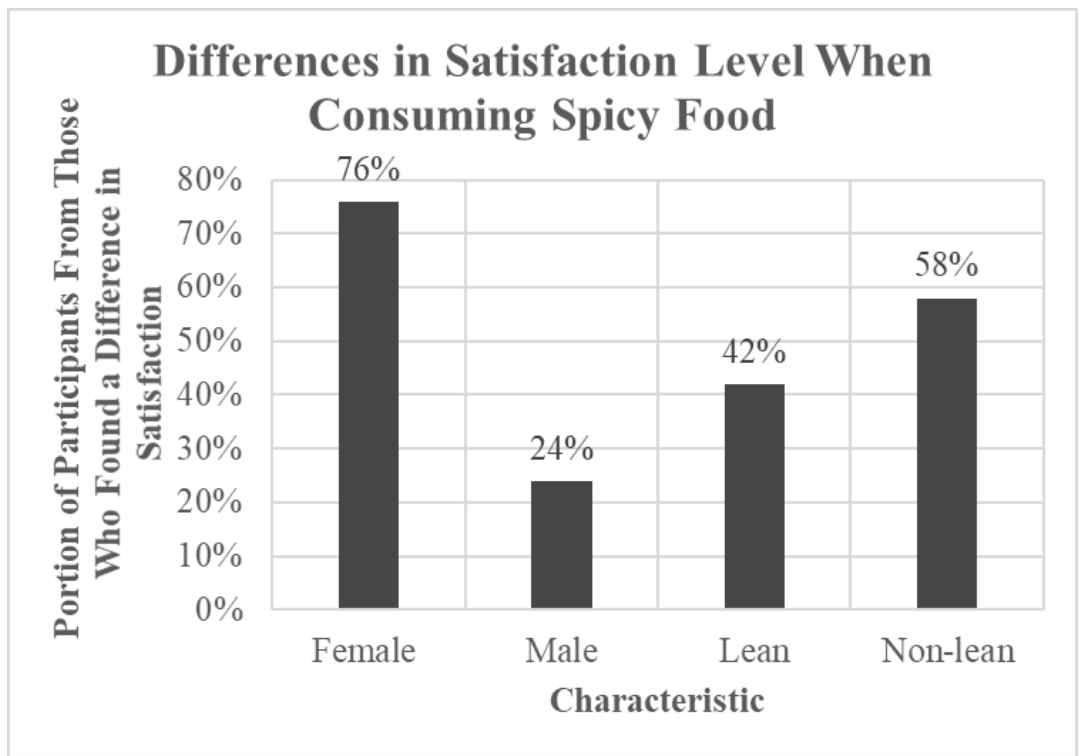

Figure 3. Bar graphs represent the percentage breakdown of male or female and lean or non-lean participants of those who found differences in satisfaction following spicy food consumption.

Most participants stated that their environment, including their family and friends, significantly influenced their choices regarding spicy foods

Table 2. Quotations directly from interviewed participants with regards to environmental influences on spicy food liking and overall consumption.

\begin{tabular}{|c|l|}
\hline Subject Number & \multicolumn{1}{c|}{ Quotation from Transcript } \\
\hline 9 & $\begin{array}{l}\text { "Totally influenced because my husband cooks and he's the spicy guy. He does come } \\
\text { from a Hispanic background." }\end{array}$ \\
\hline 52 & $\begin{array}{l}\text { "My dad liked hot peppers and so I learned to eat those with him and enjoy them and I } \\
\text { still enjoy hot peppers, like a jar of pickled peppers." }\end{array}$ \\
\hline
\end{tabular}


Most participants stated that they ate the most amount of spicy food when they were at home which consisted of $33.33 \%$ of participants. The next highest category was people who ate most of their spicy food out of their home which consisted of $25.00 \%$ of participants.

Participants were asked if they used hot sauce on food and how often they used it. $58.33 \%$ of individuals used hot sauce while $40.00 \%$ did not. Representatively, $71.43 \% \quad(z=-0.733$, $p=0.2317792$ ) of those who used hot sauce were female, $57.14 \%(z=0.648, p=0.2584925)$ were non-lean, and $42.86 \%(z=-$ $0.648, p=0.2584925)$ were lean.
A large portion including $68.33 \%$ of participants stated that the social-based, environmental influence of family and friends played a role in choices regarding spicy foods. Of those, $41.46 \%$ stated that it had a very large effect on their personal food choices leaving only $26.67 \%$ to say that it plays no role on whether spicy food is consumed in increased or decreased amounts. Genetic factors have been found to play a role between $18 \%$ and $58 \%$ (Tornwall et al., 2012), but the remainder of variation found in spicy food tolerance was due to environmental factors. $87.50 \%(z=2.860, p=0.0021182)$ of those who said family and friends played no role in their food choices regarding spicy food were non-lean.

\section{Level of Influence Environmental Factors Have on Spicy Food Consumption}

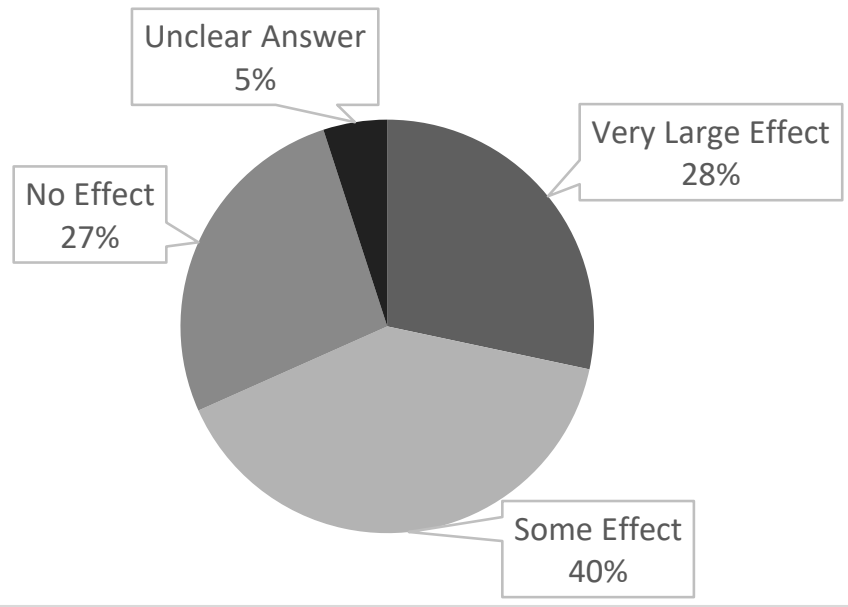

Figure 4. Pie chart represents the percentage of total survey population who stated environmental factors, such as family, friends, culture, and geographical location, influenced their levels of spicy food consumption.

\section{DISCUSSION}

\section{Population unclear on difference between "hot" and "spicy" terminology}

The study suggests the importance of understanding the difference between "hot" and "spicy" food to properly convey quantity of spicy food consumption. A great majority of the participants would say the term "hot" in the context of food could be used interchangeably with the term "spicy." Since the main purpose of this study is to determine the correlation, if any, between physical attributes and spicy food intake, it is important for the participants to fully understand the difference between "hot" and "spicy" food. Knowing the difference between the terms was especially important for answering survey questions about spicy food consumption and then relating them to lean and non-lean groups. The standard, set for this study, considered any food containing capsaicin to be considered "spicy" whereas "hot" as only a measure of temperature, but not capsaicin content. When asked if "spicy" food is the same as "hot" food, $53.00 \%$ responded that it was not meant to have the same meaning with exactly $50.00 \%$ being both lean and non-lean participants. Therefore, data from the study revealed the lack of understanding from the participants of the difference between "hot" and "spicy" foods.

\section{Food preferences driven by healthy ingredients and cost}

It has been previously found that taste and nutritional value are among the top attributes that drive decisions regarding food (Eertmans, Baeyens, \& Van den Bergh, 2001; IFIC, 2014). The results found here agree with this observation. Almost half of the participants reported that they focused on nutritional values and how healthy they determined the food to be. Almost all lean participants fully understood the ramifications of increased food intake and understood the significant direct relationship their diet has with their health. More than half of those who stated that health benefits of the food they consumed was their primary concern, were non-lean. The fact 
that a significant number of non-lean participants stated their primary concern regarding food choice was the health benefits lead us to hypothesize why they may be non-lean. Perhaps their eating habits could be linked to increased consumption which has been dramatically elevated over the recent years (Kearney, 2010). Contrarily, of the $26.67 \%$ of participants who stated that taste was the primary factor concerning food choice, $50.00 \%$ were lean and $50.00 \%$ were non-lean.

\section{Spicy food consumption increased satisfaction and decreased portion size}

The study was completed to understand how food preferences differed among certain attributes and specifically how lean and non-lean consumers differed in their choices regarding spicy food. The overwhelming majority of participants stated how foods that contain capsaicin create a burning sensation, are irritating, are not preferred compared to non-spicy food, or are simply unpleasant to consume. Only $8.33 \%$ of participants stated that they find the stimulus created by capsaicin to be pleasant and satisfying. Opposite of what has been observed previously, four of those five participants were female and three of them were non-lean which supports the trend of more non-lean individuals enjoying spicy food on a regular basis. However, since this observation is only based on three people out of the full 60 participants in this study, the data cannot be conclusive compared to the many quotes that support spicy food consumption and its correlation with increased satisfaction and smaller portion size suggesting a healthier, leaner lifestyle. A 63.33\% participant group expressed a greater sense of satisfaction after eating a meal that had spices containing capsaicin. This was much different from the remaining group of $36.67 \%$ of participants who found either no difference in satisfaction or a decrease in satisfaction when consuming spicy food compared to food that did not contain capsaicin. Of those who found increased satisfaction, $76.00 \%$ were females suggesting that spicy food has no difference in impact on females when compared to males. In contrast it has been found that males have a greater tendency to consume spicy food due to increased endogenous testosterone (Bègue et al., 2015).

The largest group of $51.67 \%$ of participants stated they would eat less spicy food if it were served compared to the next largest group of $31.67 \%$ of participants who stated it would have no effect on their levels of consumption. Another $11.67 \%$ of participants said they would eat more spicy food if it were served. The major theme of this data can be summarized by a quote from participant \#47. They stated, "I think I can feel fuller faster when I'm eating spicy hot foods then when I'm just eating sort of, I'm going to say a bland meal."

\section{External forces play a role in spicy food consumption on a daily basis}

The group that stated they consumed the spiciest food when at home was the largest compared to eating spicy food elsewhere such as restaurants. The participant's preference for where they eat the highest amount of spicy food may suggest the most comfortable and familiar environment, such as at home. A large portion of participants that made up $58.33 \%$ of the total participants stated they used various hot sauces daily. There was slight difference between the percentage of males and females who enjoyed utilizing sauce to make their meals spicy. Males were more likely to use hot sauce which follows past trends of sensation seeking individuals and increased endogenous testosterone (Bègue et al., 2015; Byrnes \& Hayes, 2013; Zuckerman et al., 1964). Environmental factors, such as the influence of family, friends, culture, and geographical location, were found to have a huge effect on personal choices regarding food. Participant \#9 stated, "Totally influenced because my husband cooks and he's the spicy guy. He does come from a Hispanic background." A large portion of participants consisting of $68.33 \%$ of the total 60 participants said family and friends influenced spicy food consumption. Another $28.33 \%$ of those participants went on to say that the influence was very large. The most significant finding that supports non-lean populations being correlated to their lack of consuming spicy food was observed where $87.50 \%$ of those who said family and friends did not influence them to consume spicy food were non-lean.

\section{CONCLUSIONS}

The subcategory of age proved to have no correlation with the amount or type of food consumed whether that be spicy or non-spicy. While males representatively did consume more spicy foods than females, overall, more lean participants stated they consumed spicy food regularly compared to non-lean participants. This data suggests that spicy food preference could be related to the status of lean versus non-lean in which decreased spicy food consumption shows a correlation with those who are subcategorized as non-lean.

Conclusions from the study provide a basic understanding of food preferences from a relatively large population study. Additional support can also be provided to quantitative scientific research on lipid metabolism activation through the qualitative aspect completed here. Few limitations were present during this survey-based study including a large female to male participant ratio making extrapolation of findings more difficult.

The study provides data to create a larger nationwide survey to assess and validate study results. Results from the data reveal how approximately only half of individuals understand the difference between "hot" and "spicy" and how a state of being lean shares a positive correlation with spicy food consumption. The long-term impact of this study is predicted to provide the fundamental information to move the study of capsaicin in obesity management toward the development and commercialization of a safe and effective food supplement to address the major health issue of obesity. 


\section{Appendix}

QUAN Data Collection

Product: Categorical responses

- Institutions'

demographic

information

- Quantitative responses

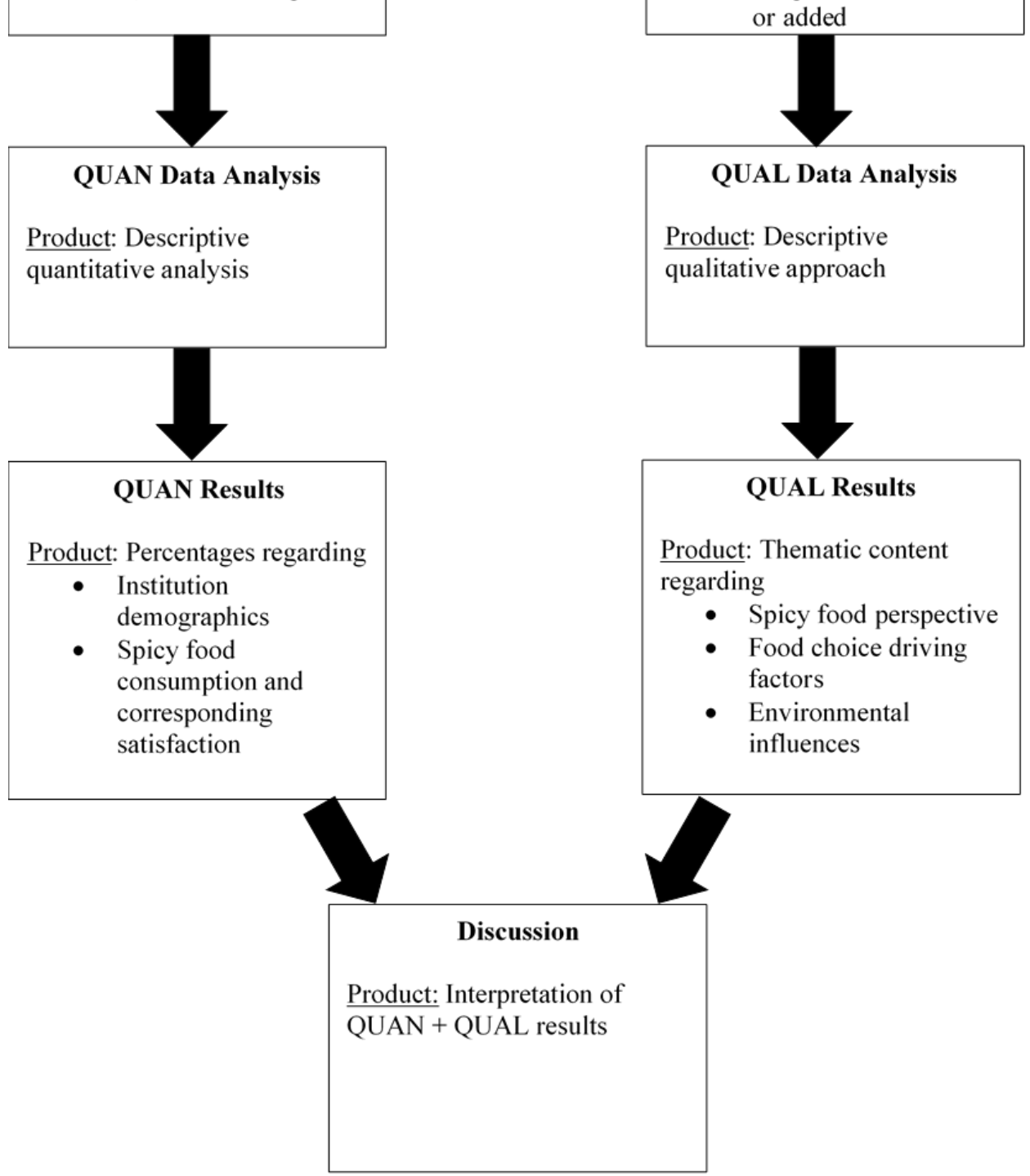

QUAL Data Collection

Product: Textual responses

- Responses to designated open-ended items

- Responses written in or added

QUAL Data Analysis

roduct: Descriptive ualitative approach

Product: Thematic content arding

Spicy food perspective Food choice driving Environmental influences

Figure A.1. Concurrent mixed methods study design. 


\section{Describe the most important factor in your choice of a food item.}

2. What is your definition of "hot" in the context of food?

3. Is "spicy" food the same as "hot" food? Why or why not?

4. Do you eat hot or spicy foods? How often? Why?

5. Where do you eat spicy foods most?

6. If you do not eat hot or spicy foods, why not?

7. If you eat spicy food, describe its effect.

8. Any differences in your sense of satisfaction when eating spicy food?

9. Is there any difference in the amount of food consumed- spicy versus non-spicy? Overall? Portion size?

10. Do you use hot sauce on food? How often? What kinds?

11. What influence do your family and friends have on your choices regarding spicy foods?

Figure A.2. Interview questions.

\section{Funding}

Research reported in this publication was supported by the Mountain West Clinical Translational Research - Infrastructure Network under a grant from National Institute of General Medical Sciences of the National Institutes of Health under Award Number IU54GM104944. The content is solely the responsibility of the authors and does not necessarily represent the official views of the National Institute of Health.

\section{Acknowledgements}

The authors of this paper would like to thank all the participants for their time and help with this study's interviews. We would also like to thank student Megan Meier, Pharm.D. candidate for her collaboration and support as well as Lawrence Staubach, M.D., M.B.A for his help with data collection.

\section{REFERENCES}

Baskaran, P., Covington, K., Bennis, J., Mohandass, A., Lehmann, T., \& Thyagarajan, B. (2018). Binding Efficacy and Thermogenic Efficiency of Pungent and Nonpungent Analogs of Capsaicin. Molecules, 1-11. https://doi.org/10.3390/molecules23123198

Bègue, L., Bricout, V., Boudesseul, J., Shankl, R., \& Duke, A. A. (2015). Some like it hot: Testosterone predicts laboratory eating behavior of spicy food. Physiology \& Behavior. 139, 375-377. https://www.sciencedirect.com/science/article/pii/S0031938414 005940

Bloomer, R. J., Canale, R. E., Shastri, S., \& Suvarnapathki, S. (2010). Effect of oral intake of capsaicinoid beadlets on catecholamine secretion and blood markers of lipolysis in healthy adults : a randomized, placebo controlled, double-blind, crossover study. Lipids Health Dis, 1-7. https://doi.org/10.1186/1476$511 X-9-72$

Byrnes, N.K., \& Hayes, J.E. (2013). Personality factors predict spicy food liking and intake. Food Quality and Preference. 28, 213-221. https://www.ncbi.nlm.nih.gov/pubmed/23538555

Byrnes, N.K., \& Hayes, J.E. (2015). Gender differences in the influence of personality traits on spicy food liking and intake. Food Quality and Preference. 42, 12-19.

https://www.ncbi.nlm.nih.gov/pmc/articles/PMC4316214/ 
Caterina, M. J., Schumacher, M. A., Tominaga, M., Rosen, T. A., Levine, J. D., \& Julius, D. (1997). The capsaicin receptor : a heatactivated ion channel in the pain pathway. Nature, 389(October). https://doi.org/10.1038/39807

Cowart, B.J. (1981). Development of taste perception in humans: sensitivity and preference throughout the life span. Psychological Bulletin. 90, 43-73.

https://www.ncbi.nlm.nih.gov/pubmed/7267897

Eertmans, A., Baeyens, F., \& Van den Bergh, O. (2001). Food likes and their relative importance in human eating behavior: review and preliminary suggestions for health promotion. Health Education Research. 16, 443-456.

https://www.ncbi.nlm.nih.gov/pubmed/11525391\%20

Green, B. G., \& Hayes, J.E. (2003). Capsaicin as a probe of the relationship between bitter taste and chemesthesis. Physiology \& Behavior. 79, 811-821.

https://www.ncbi.nlm.nih.gov/pubmed/12954427

Haramizu, S., Kawabata, F., Masuda, Y., Ohnuki, K., Watanabe, T., Yazawa, S., \& Fushiki, T. (2011). Capsinoids, non-pungent capsaicin analogs, reduce body fat accumulation without weight rebound unlike dietary restriction in mice. Bioscience,

Biotechnology, and Biochemistry. 75, 95-99.

https://www.ncbi.nlm.nih.gov/pubmed/21228478

Haramizu, S., Kawabata, F., Ohnuki, K., Inoue, N., Watanabe, T., Yazawa, S., \& Fushiki, T. (2011). Capsiate, a non-pungent capsaicin analog, reduces body fat without weight rebound like swimming exercise in mice. Biomedical Research. 32, 279-284.

https://www.ncbi.nlm.nih.gov/pubmed/21878735

IFIC (2014). Food \& Health Survey: Consumer attitudes toward food safety, nutrition, and health. Washington, DC: International Food Information Council Foundation. 90.

https://www.foodinsight.org/sites/default/files/FINAL\%202014\% 20Food\%20and\%20Health\%20Survey\%20Executive\%20Summary_ $0 . p d f$

Janssens, P.L., Hursel, R., Martens, E.A., \& Westerterp-Plantenga, M.S. (2013). Acute Effects of Capsaicin on Energy Expenditure and Fat Oxidation in Negative Energy Balance. Public Library of Science One. 8, e67786.

https://www.ncbi.nlm.nih.gov/pubmed/23844093

Karrer, T. \& Bartoshuk, L. (1991). Capsaicin desensitization and recovery on the human tongue. Physiology and Behavior. 49, 757764.

https://www.ncbi.nlm.nih.gov/pubmed/1881981

Kearney, J. (2010). Food consumption trends and drivers.

Philosophical Transactions of the Royal Society B. 365, 2793-2807.

https://www.ncbi.nlm.nih.gov/pmc/articles/PMC2935122/
Lawless, H., Rozin, P., \& Shenker, J. (1985). Effects of oral capsaicin on gustatory, olfactory and irritant sensations and flavor identification in humans who regularly or rarely consume chili pepper. Chemical Senses. 10, 579-589. http://psycnet.apa.org/record/1987-17636-001

Logue, A. W. \& Smith, M. E. (1986). Predictors of food preferences in adult humans. Appetite. 7, 109-125.

https://www.ncbi.nlm.nih.gov/pubmed/3740828

Ludy, M.J. \& Mattes, R.D. (2011). The effects of hedonically acceptable red pepper doses on thermogenesis and appetite. Physiology and Behavior. 102, 251-258.

https://www.ncbi.nlm.nih.gov/pubmed/21093467

Ludy, M.J., Moore, G.E., \& Mattes, R.D. (2012). The effects of capsaicin and capsiate on energy balance: critical review and meta-analyses of studies in humans. Chemical Senses. 37, 103121.

https://www.ncbi.nlm.nih.gov/pubmed/22038945

Ludy, M.J. \& Mattes, R.D. (2012). Comparison of sensory, physiological, personality, and cultural attributes in regular spicy food users and non-users. Appetite. 58, 19-27. https://ac.elscdn.com/S0195666311005861/1-s2.0-S0195666311005861main.pdf?_tid=d1245178-2186-4d85-a34b78f800d18b29\&acdnat=1541459095_5edbd8858f268604a6b392 12b07de88a

Mitchell, N., Catenacci, V., Wyatt, H. R., \& Hill, J. O. (2011). Obesity: Overview of an Epidemic. Psychiatric Clinics of North America. 34, 717-732.

https://www.ncbi.nlm.nih.gov/pmc/articles/PMC3228640/

Nirengi, S. (2016). Assessment of human brown adipose tissue density during daily ingestion of thermogenic capsinoids using near-infrared time-resolved spectroscopy. J Biomed Opt, 21(9). https://doi.org/10.1117/1.JBO.21.9.091305

Ohnuki, K., Haramizu, S., Oki, K., Watanabe, T., Yazawa, S., \& Fushiki, T. (2001). Administration of capsiate, a non-pungent capsaicin analog, promotes energy metabolism and suppresses body fat accumulation in mice. Bioscience, Biotechnology, and Biochemistry. 65, 2735-2740.

https://www.ncbi.nlm.nih.gov/pubmed/11826971

Rondanelli, M., Opizzi, A., Perna, S., Faliva, M., Solerte, S. B., Fioravanti, M., ... Solerte, S. B. (2013). Journal of the American College of Nutrition Acute Effect on Satiety, Resting Energy Expenditure , Respiratory Quotient, Glucagon-Like Peptide-1, Free Fatty Acids, and Glycerol Following Consumption of a Combination of Bioactive Food Ingredients in Ov. J Am Coll Nutr, 5724. https://doi.org/10.1080/07315724.2013.767667

Rozin, P. \& Schiller, D. (1980). The nature and acquisition of a preference for chili pepper by humans. Motivation and Emotion. 4, 77-101.

https://link.springer.com/article/10.1007/BF00995932 
Singh, R., Staubach, L., Frantz, J., \& Thyagarajan, B. (2017).

Understanding Food Choice, Medication Use, and Health from Lean and Non-Lean Resident Perspectives in a Rural State. BAOJ

Pharmaceutical Sciences. 3, 1-8.

https://bioaccent.org/pharmaceutical/pharmaceutical34.pdf1

Stevenson, R. J. \& Prescott, J. (1994). The effects of prior

experience with capsaicin on ratings of its burn. Chemical Senses.

19, 651-656.

https://www.ncbi.nlm.nih.gov/pubmed/7735844

Stevenson, R. J. \& Yeomans, M. R. (1995). Does exposure enhance liking for the chilli burn? Appetite. 24, 107-120. https://ac.elscdn.com/S0195666395993282/1-s2.0-S0195666395993282main.pdf?_tid=116f6ab5-9c98-4ec9-bdcdaee6ac2b0f2c\&acdnat=1541461652_79c8d0f192a8bd28fd991618 2901a9e0

Tornwall, O., Silventoinen, K., Kaprio, J., \& Tuorila H. (2012). Why do some like it hot? Genetic and environmental contributions to the pleasantness of oral pungency. Physiology \& Behavior. 107, 381-389. https://www.ncbi.nlm.nih.gov/pubmed/23010089

Xue, Y., He, T., Yu, K., Zhao, A., Zheng, W., Zhang, Y., \& Zhu, B. (2017). Association between spicy food consumption and lipid profiles in adults: a nationwide population-based study. British Journal of Nutrition. 118, 144-153.

https://www.ncbi.nlm.nih.gov/pubmed/28673367

Zuckerman, M., Kolin, E. A., Price, L., \& Zoob, I. (1964).

Development of a sensation-seeking scale. Journal of Consulting

Psychology. 28, 477-482.

http://psycnet.apa.org/record/1965-07735-001 